\title{
Cation diffusion in alkaline-earth oxides
}

\author{
J H Harding $\dagger$, M J L Sangster $\ddagger$ and A M Stoneham $\dagger$ \\ $\uparrow$ Theoretical Physics Division, B424.4, Atomic Energy Research Establishment, \\ Harwell, Didcot, Oxfordshire, UK \\ $\ddagger$ J J Thomson Physical Laboratory, University of Reading, Whiteknights, Reading, UK
}

Received 30 March 1987

\begin{abstract}
Absolute jump rates for cation-vacancy interchanges in $\mathrm{MgO}, \mathrm{CaO}, \mathrm{SrO}$ and $\mathrm{BaO}$ are calculated from a set of model inter-ionic potentials. Internal energies and vibrational entropies over a wide range of temperatures (i.e. at expansions which within the models correspond to these temperatures in the quasi-harmonic approximation) are evaluated and, from these, migration enthalpies and pre-exponential frequency factors are deduced. Correlations between these two diffusion parameters for the family of oxides are investigated.
\end{abstract}

\section{Introduction}

Calculations based on inter-atomic potential models of internal energies of defects in crystals have been rendered routine by the development of computer codes such as HADES (Norgett 1974, see also Catlow and Mackrodt 1982). More recently, general codes have been written for the calculation of vibrational entropies again from interatomic potential models. One method, the large-crystallite method, in which explicit vibrations of atoms within a spherical region surrounded by a rigid outer region are considered, may be considered as a dynamic counterpart of the Mott-Littleton (1938) approach employed in the HADES package. Details of a computer code (SHEOL) based on this method have been given by Harding (1985a). With general-purpose computer packages available for both internal energies and entropies, it is now possible to make accurate numerical investigations of the thermodynamics of defective crystals described in terms of inter-atomic interaction potentials. A number of such studies have now appeared, e.g. the work by Harding (1985b) on defect free energies in calcium fluoride.

In this paper, we apply the above methods to an investigation of cation diffusion in the alkaline-earth oxides, based on the potential models of Stoneham and Sangster (1985) although, as discussed in $\S 3.1$, some modifications are required in the description of $\mathrm{BaO}$ to avoid a dielectric catastrophe as the crystal is expanded. A similar investigation of cation-vacancy jumps in $\mathrm{MgO}$ has been reported earlier by Sangster and Stoneham (1984) and, in this case, direct comparison with the experimental work of Sempolinski and Kingery (1980) can be made. For the other oxides, no experimental measurements are available and therefore our calculations of activation enthalpies and pre-exponential frequency factors are purely predictive. 
The main reason for choosing the alkaline-earth oxides as the object of our study was to have a set of host crystals which could reasonably be taken to constitute a 'family' (in the sense that properties for different members can possibly be related by some simple scaling factors) while at the same time displaying an extended range of values for these properties (e.g. lattice parameter, expansion coefficients and lattice vibrational frequencies). By contrast a family of transition-metal oxides would, at least on the basis of most potential models, show a very small spread in such values. We shall, in section 4 , investigate correlations between the model activation enthalpies and frequency factors for the family. Empirical correlations of this type have been noted for jump processes in many sets of related systems and are generally described as either a compensation rule or a Meyer-Neldel (1937) rule. To test whether or not these rules are satisfied by our modelled oxides, it is clearly desirable to have a large variation across the family of both the enthalpies and the frequency factors.

In $\S 2$ the necessary steps in the calculations are summarised and the methods of computation are briefly discussed. Our results are presented in $\S 3$ and finally, in $\S 4$, the correlations are investigated. We find that, within the numerical uncertainties of our calculated values, the pre-exponential frequency factor being subject to considerable inaccuracy, a Meyer-Neldel type of correlation is satisfied. We also find that, from the energy scaling implied by the activation enthalpies, reasonable values for the preexponential frequency factors may be deduced when account is taken of differences in cation mass and lattice spacing. Furthermore, the same scaling arguments provide good estimates of the ratios of frequencies of normal modes for the host lattice in which only the cations are displaced. This suggests a number of methods for estimating the parameters for cation diffusion in the whole family of alkaline-earth oxides from detailed calculations for one member.

\section{Method of calculation}

The calculations on alkaline-earth oxides reported here parallel earlier work on $\mathrm{KCl}$ and $\mathrm{CaF}_{2}$ (Harding 1985a, b). Both the methods and the computational techniques have been fully discussed in these papers; in this section, we shall merely summarise the various steps that are required in the calculation. A useful collection and discussion of the necessary results from defect thermodynamics is to be found in a paper by Catlow et al (1981).

Jump rates for the cation-vacancy interchanges with which we are concerned may be written in a number of equivalent ways. From Vineyard's (1957) analysis the rates may be written in terms of the migration energy $u_{\mathrm{m}, v}$ :

$$
\Gamma=\tilde{\nu} \exp \left(-u_{\mathrm{m}, v} / k T\right)
$$

with $\tilde{\nu}$ an effective frequency. Alternatively, in Flynn's (1972) formulation, the rates may be expressed in terms of the free energy $f_{\mathrm{m}, V}$ as

$$
\Gamma=\nu_{0} \exp \left(-f_{\mathrm{m}, V} / k T\right)
$$

with

$$
f_{\mathrm{m}, V}=u_{\mathrm{m}, V}+k T\left[\sum_{j=1}^{3 N} \ln \left(\frac{h \nu_{j}^{\prime}}{k T}\right)-\sum_{j=1}^{3 N} \ln \left(\frac{h \nu_{j}}{k T}\right)\right]
$$


Here $\nu_{j}$ are normal mode frequencies in the initial (equilibrium) configuration and $\nu_{j}^{\prime}$ are those for the saddle-point configuration, with the imaginary frequency of the unstable mode being replaced by $\nu_{0}$. Thus, although the frequency $\nu_{0}$ appears formally in equations (2) and (3), it may be given any value.

The migration energy $u_{\mathrm{m}, V}$ is obtained as the difference in defect energies in saddlepoint and initial configurations, using the standard code HADES (Norgett 1974, 1977; see also Catlow and Mackrodt 1982) for the defect energy determinations. The remaining vibration entropy contributions to $f_{\mathrm{m}, V}$ is calculated in the SHEOL package (Harding $1985 \mathrm{~b}$ ) by finding the frequencies for normal modes of large crystallites embedded in a static dielectric continuum first with the crystallite in the saddle-point and then in the initial (equilibrium) configuration. (In order to make corrections for the effects of the long-range distortion field, first pointed out by Gillan and Jacobs (1983), normal mode frequencies are also calculated for other configurations, namely the perfect lattice with the distortions associated with the two defect configurations.) In previous work on rocksalt structure hosts (Harding 1985a, Sangster and Stoneham 1984) it was found that in addition to the unstable mode at the saddle point there was a second mode, with the principal motion being that of the diffusing ion at right angles to the jump path, which either has a low frequency or may also be unstable. In either case the harmonic approximation will not be adequate. A method for including anharmonicity for this degree of freedom has been discussed by Harding (1985a); see also Sangster and Stoneham (1984) for simpler approaches. It is assumed that only the displacement of the hopping ion is involved in the mode and an effective frequency is deduced from the mode contribution to the configurational partition function, which is evaluated by numerical integration.

Application of the above methods supplies not only $f_{\mathrm{m}, V}$ but also the migration entropy at constant volume:

$$
s_{\mathrm{m} . V}=-k\left[\sum_{j=1}^{3 N} \ln \left(\frac{h \nu_{j}^{\prime}}{k T}\right)-\sum_{j=1}^{3 N} \ln \left(\frac{h \nu_{j}}{k T}\right)\right]
$$

(i.e. the entropy contribution to equation (3)). Thus, constant-volume energies and vibrational entropies may be calculated by fairly standard means. However, experiments are always carried out at constant pressure and equation (2) must be recast in terms of experimentally accessible quantities. Making use of the relation

$$
g_{\mathrm{m}, p}=f_{\mathrm{m}, V}
$$

which is correct to first order in defect volume (Catlow et al 1981), we may write

$$
h_{\mathrm{m}, p}=f_{\mathrm{m}, V}+T s_{\mathrm{m}, p}
$$

and hence equation (2) may be re-expressed in terms which are directly accessible in (constant-pressure) experiments as

$$
\Gamma=A \exp \left(-h_{\mathrm{m}, p} / k T\right)
$$

with

$$
A=\nu_{0} \exp \left(s_{\mathrm{m}, p} / k\right) .
$$

In equation (7), $\nu_{0}$ is again the arbitrarily chosen frequency used in the vibrational entropy calculations. The result for $A$ is independent of this choice. 
Our aim is to calculate both $A$ and $h_{\mathrm{m}, p}$ in equation (7). The migration enthalpy $h_{\mathrm{m}, p}$ is calculated from equation (6) with the first term given by equation (3) (at constant volume). What is required both for completion of the calculation of $h_{\mathrm{m}, p}$ (equation (6)) and for the calculation of pre-exponential factor $A$ (equation (7)) is the corresponding entropy at constant pressure:

$$
s_{\mathrm{m}, p}=-\left(\frac{\partial}{\partial T}\left(g_{\mathrm{m}, p}\right)\right)_{p}=-\left(\frac{\partial}{\partial T}\left(f_{\mathrm{m}, V}\right)\right)_{p} .
$$

This makes use of a standard thermodynamic relation and then equation (5). The procedure used in the calculation of the entropy $s_{\mathrm{m}, p}$ is to obtain the free-energy $f_{\mathrm{m}, V}$ over a range of volumes each of which corresponds to a particular temperature (and $p=$ 0 ). These temperatures are evaluated from the model potentials, within the quasiharmonic approximation, from the balance of the elastic and vibrational pressures (Harding 1985a, b). Hence we obtain $f_{\mathrm{m}, V}(T)$ at $p=0$, and $s_{\mathrm{m}, p}$ is then given by the temperature gradient. The steps in the calculations and their interconnections are shown as a flow diagram in figure 1.

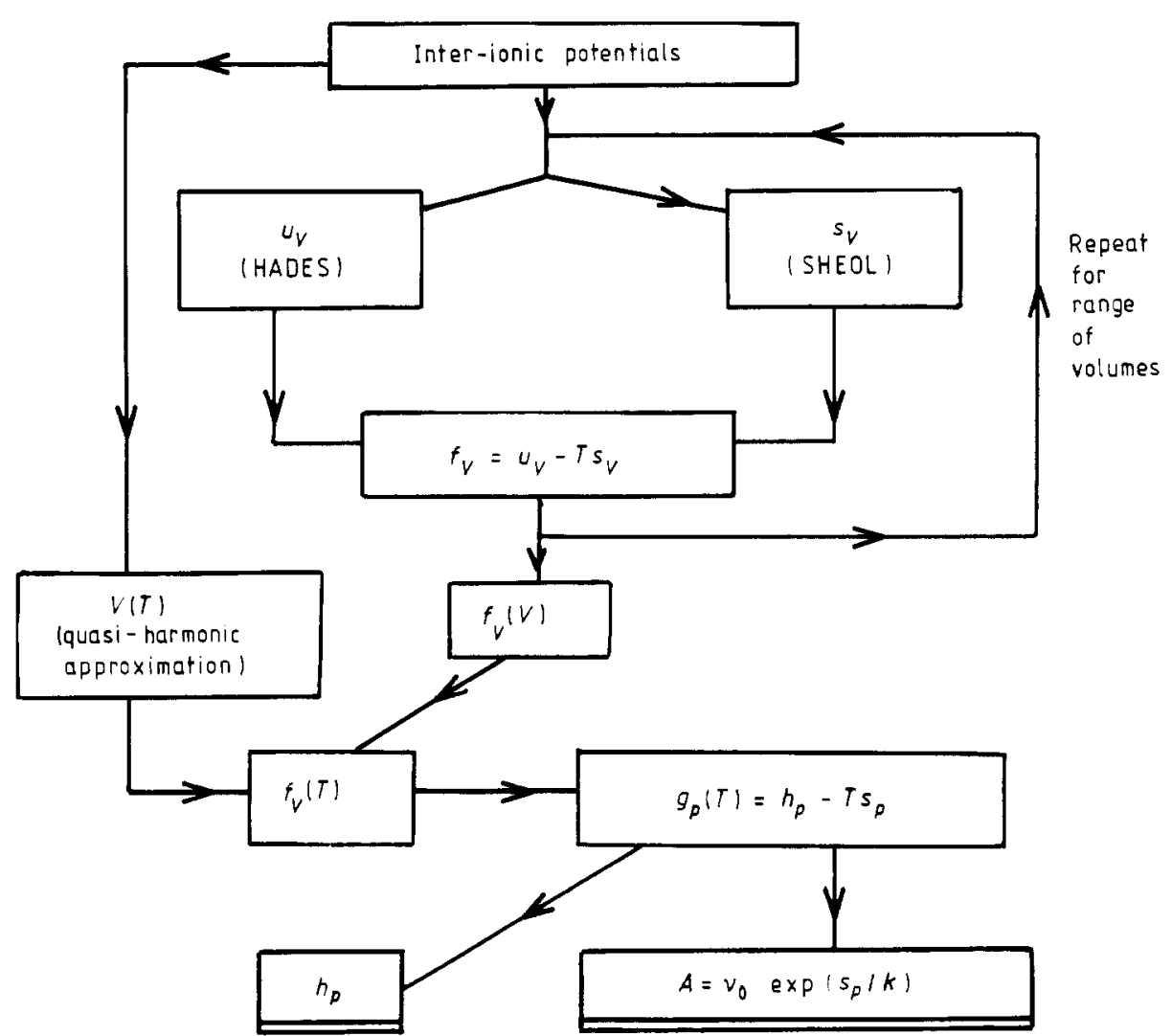

Figure 1. Diagram to show components of calculations of jump rate parameters from interionic potentials. 


\section{Numerical results}

\subsection{Lattice expansion}

The first requirement is to find the relation between lattice parameter and temperature predicted by the model potentials, namely those given by Stoneham and Sangster (1985). The compressibility at a given expansion is obtained directly from the potentials and hence the elastic pressure for any expansion can be found by numerical integration. The procedure is to chose a particular expansion, to find the frequencies of normal modes in the expanded crystal for a sample of wave-vectors and hence to determine the vibrational free energy as a function of temperature. The vibrational pressure, again as a function of temperature and for a fixed expansion, is given by the volume derivative of the free energy; the mode Grüneisen parameters are evaluated from the small changes in frequency upon introducing small volume changes. The temperature corresponding to the chosen expansion is that at which the vibrational and elastic pressures balance. Such calculations were carried out (with 148 wave-vectors in the symmetry-reduced Brillouin zone) over the anticipated range of expansions.

In all four alkaline-earth oxides, it was found that, as successive (equal) increases in lattice spacing were made, the corresponding steps in temperature decreased. For $\mathrm{BaO}$ there was a charge in sign, i.e. beyond a certain stage further expansion implies a reduction in temperature. This renders the potential model unusable for our present purposes. The reason for this instability in the model is that the static dielectric constant, which is very high (34.0) without expansion, rapidly becomes enormous as the crystal expands. This pulls the TO branches to very low frequencies with disastrous effect on the free energy. Since, as stated in $\S 1$, one of the aims of the present work requires as wide as range of crystal properties as possible, it was decided to modify the potentials rather than to abandon this extreme of our range. The static dielectric constant was artificially lowered by reducing the shell charge of $\mathrm{Ba}^{2+}$ to 3.2 , a value similar to the shell charges for $\mathrm{Ca}^{2+}$ and $\mathrm{Sr}^{2+}$ while leaving the polarisability unchanged by making a compensating change in the shell-core force constant.

With this adjustment for $\mathrm{BaO}$, but no modifications to the potentials for the other oxides or the inter-ionic terms for $\mathrm{BaO}$, the temperature dependences of the nearestneighbour distances were calculated and fitted to the quadratic

$$
a(T)=a(T=0)+\lambda_{1} T+\lambda_{2} T^{2}
$$

with the coefficients listed in table 1 . Also given are the model values for the lattice expansion at room temperature. Agreement with experimental values is rather poor, these being larger by factors of around 2 . It is important, however, to perform the

Table 1. Temperature dependence of lattice parameters: $a(T)=a(T=0)+\lambda_{1} T+\lambda_{2} T^{2}$. The final column gives the coefficient of linear expansion at room temperature.

\begin{tabular}{lllcl}
\hline & $\begin{array}{l}a(T=0) \\
(\AA)\end{array}$ & $\begin{array}{l}\lambda_{1} \\
\left(10^{-5} \AA \mathrm{K}^{-1}\right)\end{array}$ & $\begin{array}{l}\lambda_{2} \\
\left(10^{-9} \AA \mathrm{K}^{-2}\right)\end{array}$ & $\begin{array}{l}\alpha_{\mathrm{RT}} \\
\left(10^{-6} \mathrm{~K}^{-1}\right)\end{array}$ \\
\hline $\mathrm{MgO}$ & 2.1026 & 1.0663 & 4.4051 & 6.3093 \\
$\mathrm{CaO}$ & 2.4021 & 0.9563 & 9.5961 & 6.3523 \\
$\mathrm{SrO}$ & 2.5763 & 1.1001 & 14.1890 & 7.5393 \\
$\mathrm{BaO}$ & 2.7585 & 0.5376 & 25.2930 & 7.4033 \\
\hline
\end{tabular}



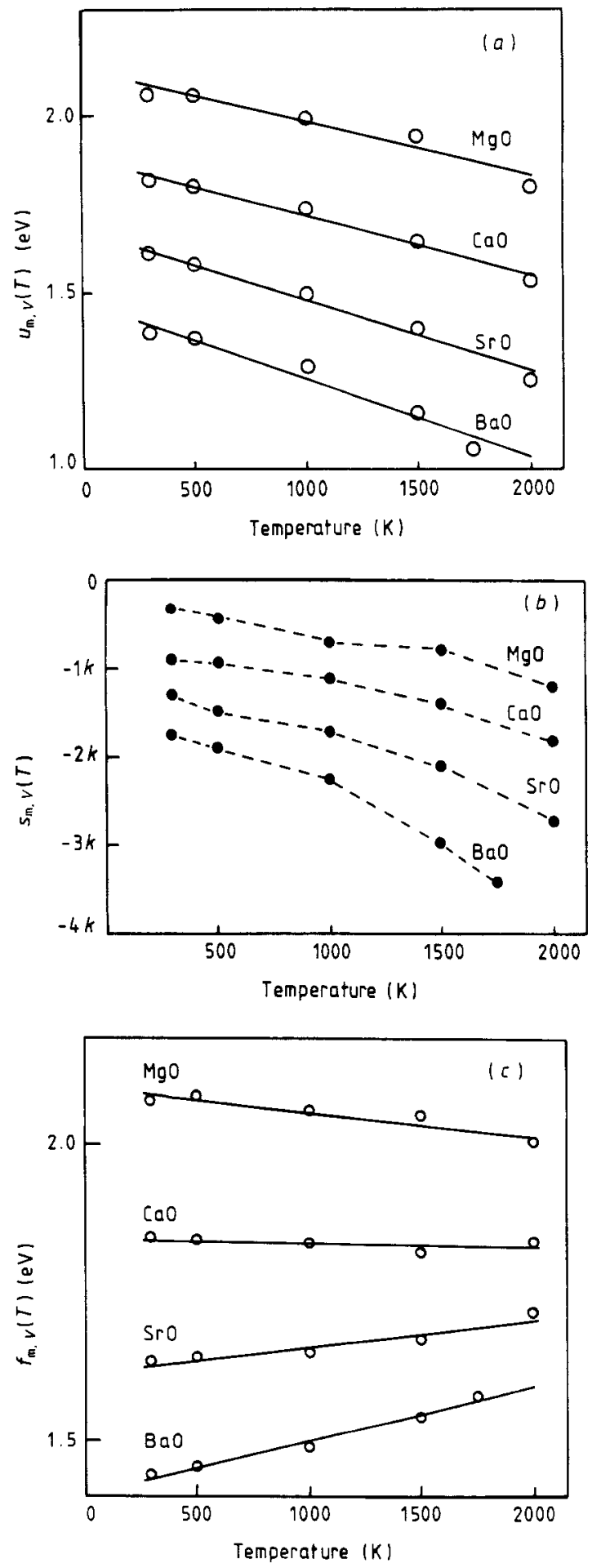

Figure 2. (a) Internal energies $u_{\mathrm{m}, V}(T)$ of migration and linear fits; (b) constant-volume entropies $s_{\mathrm{m}, v}(T)$ of migration; (c) free energies $f_{\mathrm{m}, v}(T)$ of migration at constant volume and linear fits (these are equivalent to constant-pressure Gibbs free energies $g_{m, p}(T)$ ). 
calculations entirely within the framework of the potential model and the quasi-harmonic approximation rather than to use experimental values for some parts.

\subsection{Free energies and enthalpies}

The route for obtaining the (constant-pressure) enthalpies and entropies of equations ( 7 ) and ( 8 ) has been discussed in $\$ 2$ and summarised in figure 1 . In the discussion of our results in $\S 4$, we shall also need some more detailed information on intermediate terms, particularly the constant-volume internal energies and their temperature derivatives.

For each oxide, we calculate the internal energy $u_{\mathrm{m}, v}$ of migration at fixed volumes corresponding to five temperatures between room temperature and $2000 \mathrm{~K}$ ( $1750 \mathrm{~K}$ for $\mathrm{BaO}$ which has the lowest melting point). The results are displayed as points in figure 2(a) with best-fit straight lines

$$
u_{\mathrm{m}, V}(T) \simeq a-b T \text {. }
$$

The parameters $a$ and $b$ are listed in table 2. At the same temperatures, we find the vibrational entropy $s_{\mathrm{m}, V}$ of migration and hence the free energy $f_{\mathrm{m}, V}$ following the methods in $\S 2$. The results are given as points in figures $2(b)$ and $2(c)$. Linear fits to the points in figure $2(c)$ are shown:

$$
f_{\mathrm{m}, v}(T)=g_{\mathrm{m}, p}(T) \simeq c-d T .
$$

It is doubtful whether the accuracy of the calculation is adequate to justify the use of non-linear fitting. On comparison of figures $2(a)$ and $2(c)$, it can be seen that the temperature dependence of the free energy is more linear than that of the internal energy; non-linearities in the latter are largely compensated by the additional entropy term.

Since from figure $2(c)$ these linear relations provide a reasonably good fit to the calculated points, the parameters $c$ and $d$, which are listed in table 2 , may be identified with temperature-independent constant-pressure enthalpies $h_{\mathrm{m}, p}$ of migration and entropies $s_{\mathrm{m}, p}$ of migration, respectively. This follows from the relation

$$
g_{\mathrm{m}, p}=h_{\mathrm{m}, p}-T s_{\mathrm{m}, p} .
$$

It should be recalled that at this stage all entropies include additive constants arising from the choice made for the arbitrary frequency $\nu_{0}$; we have taken $\nu_{0}$ equal to $19.6 \mathrm{THz}$, a suitable frequency for $\mathrm{MgO}$, in all cases. This arbitrariness is removed when we use equation (8) to form the pre-exponential factors $A$ of the jump rates. Table 2 includes these pre-factors.

Table 2. Parameters in linear fits to $u_{\mathrm{m} . v}(T)$ (equation (11)) and $g_{\mathrm{m} . p}(T)$ (equation (12)) and pre-factor $A$ from equation (8).

\begin{tabular}{llllllr}
\hline & \multicolumn{3}{c}{$u_{\mathrm{m}, V}=a-b T$} & & \multicolumn{3}{c}{$g_{\mathrm{m}, p}=c-d T$} \\
\cline { 2 - 3 } \cline { 5 - 6 } & $\begin{array}{l}a \\
(\mathrm{eV})\end{array}$ & $\begin{array}{l}b \\
\left(\mathrm{meV} \mathrm{K}^{-1}\right)\end{array}$ & & $\begin{array}{l}c \equiv h_{\mathrm{m}, p} \\
(\mathrm{eV})\end{array}$ & $\begin{array}{l}d \equiv s_{\mathrm{m}, p} \\
\left(\mathrm{meV} \mathrm{K}^{-1}\right)\end{array}$ & \multicolumn{1}{l}{$\begin{array}{l}A \\
(\mathrm{THz})\end{array}$} \\
\hline $\mathrm{MgO}$ & 2.140 & 0.1528 & & 2.100 & 0.04055 & 31.29 \\
$\mathrm{CaO}$ & 1.886 & 0.1698 & & 1.841 & 0.00410 & 20.55 \\
$\mathrm{SrO}$ & 1.680 & 0.2021 & & 1.615 & -0.04546 & 11.60 \\
$\mathrm{BaO}$ & 1.478 & 0.2219 & & 1.403 & -0.09556 & 6.51 \\
\hline
\end{tabular}


The counterpart of the room-temperature pre-exponential factor for $\mathrm{MgO}$ obtained from Vineyard's theory by Sangster and Stoneham (1984) is $\nu_{0} \exp \left(s_{\mathrm{m}, V} / k\right)$ rather than $A$. Taking the room temperature value for $s_{\mathrm{m}, V}$ from figure $2(b)$, we obtain a preexponential factor of $14.5 \mathrm{THz}$. The value given by Sangster and Stoneham was $32.9 \mathrm{THz}$ or around $23 \mathrm{THz}$ when adjustments for anharmonicity were made; since similar corrections for anharmonicity are made here, it is the lower value which should be compared with $14.5 \mathrm{THz}$. The main source of the discrepancy is likely to be the inaccuracies of the earlier calculation arising from the use of the supercell method with a small 64-site cell.

\section{Interpretations of results}

\subsection{Comparison with experiment and previous work}

The results on model systems given in $\$ 3$ may be used to check whether some commonly made assumptions are justified and whether or not certain empirical relationships between pre-exponential factors and enthalpies of migration hold. Only for $\mathrm{MgO}$ are any experimental values available for comparison. Sempolinski and Kingery (1980) give a measurement of $h_{\mathrm{m}, p}$ in $\mathrm{MgO}$ as $2.28 \pm 0.2 \mathrm{eV}$, a range which includes our calculated value. From their measurements of the mobility for vacancy motion (in the same paper), Sangster and Stoneham (1984) have deduced that the measured pre-exponential factor is $210 \pm 80 \mathrm{THz}$, considerably higher than the value found here. Sangster and Stoneham tried to resolve this discrepancy by adjusting the pre-exponential factor by an entropy term arising from the variation in the internal energy of migration with temperature, i.e. multiplying by $\exp (b / k)$ with $b$ as in equation (11) and table 2. Two main assumptions are involved. The first is widely made and consists of replacing $h_{\mathrm{m}, p}$ (at all temperatures) by $u_{\mathrm{m}, V}(T=0)$, i.e. $a$ in equation (11) and table 2 . Comparison of the first and third columns of the table shows that no serious error is made by this assumption. The second assumption is that the temperature variations in vibrational entropy contributions to the pre-factor are negligible; from figure $2(b)$, it can be seen that this is not justified.

Nevertheless, it is interesting to note that, when absolute rates are compared with experiment (in the temperature range of the latter), both the present and the earlier predictions are fairly close to the result of Sempolinski and Kingery (1980), $\Gamma_{\exp }=$ $210 \exp (-2.28 \mathrm{eV} / k T)=2.84 \times 10^{7} \mathrm{~s}^{-1}$ at $1400^{\circ} \mathrm{C}$. Substituting our values in equation (7) gives $\Gamma=1.48 \times 10^{7} \mathrm{~s}^{-1}$ at $1400^{\circ} \mathrm{C}$ and taking $\tilde{\nu}=23 \mathrm{THz}$ and $u_{\mathrm{m}}=1.9 \mathrm{eV}$ (at $1400^{\circ} \mathrm{C}$ ) from Sangster and Stoneham (1984, equation (1)) gives $\Gamma=4.34 \times 10^{7} \mathrm{~s}^{-1}$.

\subsection{Scaling arguments}

From the final column of table 2 , it is clear that the pre-exponential frequency $A$ decreases as the mass of the jumping ion increases. This is to be expected since the host lattice frequencies decrease in the same way. Perhaps the most relevant lattice frequency for comparison is the transverse acoustic frequency $\mathrm{TA}(\mathrm{L})$ at the $\mathrm{L}$ point. The $\mathrm{L}$ point is chosen since, for this wave-vector, one sublattice is stationary. In one of the two (degenerate) TA(L) modes the cations will be displaced in the (110) direction, which is the direction required for the jump process that we are considering. These host lattice frequencies, as obtained from our model, are listed in first column of table 3 and may be related to each other by the following simple scaling argument.

We consider a model in which the energies of all configurations for one host are obtained from those for another by a single scaling factor $\alpha$ which we take to be the ratio 
Table 3. Characteristic frequencies $\nu_{\mathrm{TA}(\mathrm{L})}$ and $\bar{\nu}_{\mathrm{RT}}$ and pre-exponential factors $A . \alpha, \beta$ and $\gamma$ are scaling ratios and the estimates found by scaling $\mathrm{MgO}$ results are given in brackets.

\begin{tabular}{|c|c|c|c|c|c|c|}
\hline & $\begin{array}{l}\nu_{\mathrm{TA}(\mathrm{L})} \\
(\mathrm{THz})\end{array}$ & $\begin{array}{l}\text { Energy } \\
\text { ratio } \\
\alpha\end{array}$ & $\begin{array}{l}\text { Lattice } \\
\text { parameter } \\
\text { ratio } \\
\beta\end{array}$ & $\begin{array}{l}\text { Cation } \\
\text { mass } \\
\text { ratio } \\
\gamma\end{array}$ & $\begin{array}{l}\ddot{\nu}_{R T} \\
(\mathrm{THz})\end{array}$ & $\begin{array}{l}A \\
(\mathrm{THz})\end{array}$ \\
\hline $\mathrm{MgO}$ & 8.69 & (1) & (1) & (1) & 14.52 & 31.29 \\
\hline $\mathrm{CaO}$ & $5.45[5.56]$ & 0.88 & 1.142 & 1.648 & $7.97[9.29]$ & 20.55 [19.98] \\
\hline $\mathrm{SrO}$ & $3.17[3.28]$ & 0.77 & 1.225 & 3.603 & $5.34[5.48]$ & $11.60[11.80]$ \\
\hline $\mathrm{BaO}$ & $2.16[2.28]$ & 0.67 & 1.311 & 5.647 & $3.37[3.81]$ & $6.51[8.21]$ \\
\hline
\end{tabular}

of migration enthalpies from table 2. Thus, for example, we assume that the energies along corresponding trajectories in two of the crystals differ only in their energy scale and their length scale $\beta$, which is the ratio of the host lattice parameters. In particular, at a minimum point on a trajectory the harmonic restoring force constant given by the curvature of the energy will scale as $\alpha / \beta^{2}$ and hence the vibrational frequency will scale as $\alpha^{1 / 2} \beta^{-1} \gamma^{-1 / 2}$ where $\gamma$ is the ratio of the cation masses. (The mass ratio is the dominant factor.) The ratios $\alpha, \beta$ and $\gamma$ taken relative to $\mathrm{MgO}$ are given in table 3 , as are the scaled $\mathrm{TA}(\mathrm{L})$ frequencies (in brackets) found by multiplying the results for $\mathrm{MgO}$ by the above scale factor. The agreement is quite satisfactory.

Although having the dimension of frequency, the pre-exponential factor $A$ is not simply related to the energy surface curvature. The scaling arguments may be more reasonably tested on the frequencies $\bar{\nu}$ in equation (1). As in $\S 3, \tilde{\nu}$ at a particular temperature is obtained from our calculations as $\nu_{0} \exp \left(s_{\mathrm{m}, V} / k\right)$ with $\mathrm{S}_{\mathrm{m}, V}(T)$ given in figure $2(b)$ (and $\nu_{0}$ fixed at $19.6 \mathrm{THz}$ ). Room-temperature values $\tilde{\nu}_{\mathrm{RT}}$ are listed in table 3 together with frequencies scaled to the $\mathrm{MgO}$ result in brackets. Again the scaled frequencies give moderately good estimates since the frequency ratio $\tilde{\nu}_{\mathrm{RT}} / \nu_{\mathrm{TA}(\mathrm{L})}$ is fairly constant over the family.

The results from applying the scaling arguments directly to $A$ are also given in table 3 and again fair agreement is found with the full calculations. This is somewhat surprising since, as pointed out above, a simple relation with the curvature of energy surfaces is not to be expected. In equation (8) the arbitrary frequency may be replaced by $\bar{\nu}_{\mathrm{RT}}$ (taking the appropriate value for each oxide) provided that corresponding adjustments to the entropy are made. Thus, we write

$$
A=\tilde{\nu}_{\mathrm{RT}} \exp \left(s_{\mathrm{m}, p}^{\mathrm{RT}} / k\right)
$$

with

$$
s_{\mathrm{m}, p}^{\mathrm{RT}}=s_{\mathrm{m}, p}+k \ln \left(\nu_{0} / \tilde{\nu}_{\mathrm{RT}}\right)=s_{\mathrm{m}, p}-s_{\mathrm{m}, V}^{\mathrm{RT}} .
$$

Note that the superscript in $s_{\mathrm{m}, p}^{\mathrm{RT}}$ does not imply a temperature dependence; it merely implies the choice of $\tilde{\nu}_{\mathrm{RT}}$ as the arbitrary frequency in the entropy calculations. (The term $s_{\mathrm{m}, p}$ without a superscript is calculated with $19.6 \mathrm{THz}$ as the arbitrary frequency as elsewhere in this paper.) It is the fact that these entropy factors, $\exp \left(s_{\mathrm{m}, p}^{\mathrm{RT}} / k\right)$ (obtained simply by equation (13) as the ratio $A / \tilde{\nu}_{\mathrm{RT}}$ ), show little variation over the family that leads to the scaling arguments giving reasonably good values for $A$. The lack of variation 
in $s_{\mathrm{m}, p}^{\mathrm{RT}}$ indicates that the dominant part of the crystal dependence of $s_{\mathrm{m}, p}$, as in table 2, is from the changes in mode frequencies and these in turn depend most critically on the mass of the hopping atom.

These arguments show that, at least for this family of crystals, if jump rates are calculated from equation (1) taking $\bar{\nu}$ as a constant multiple of a relevant characteristic lattice frequency, in our case about $3.6 \nu_{\mathrm{TA}(\mathrm{L})}$, then pre-exponential factors in fair accord with those from full calculations result. This is reassuring from the point of view that in intrinsic diffusion any frequency for attempts at barrier crossing might be expected to be simply related to typical host lattice frequencies. However, if it is assumed that the entropy factor is dominated by the temperature dependence of the internal energy, as in the earlier work by Sangster and Stoneham (1984), i.e. if we write

$$
\Gamma=\bar{\nu}^{\prime} \exp (b / k) \exp (-a / k T)
$$

with $a$ and $b$ as in equation (11), then there is a systematic variation in the entropy term $\exp (b / k)$, with the value for $\mathrm{BaO}$ being more than double that for $\mathrm{MgO}$. The main reason for the difference in behaviour between $\exp \left(s_{\mathrm{m}, p}^{\mathrm{RT}} / k\right)$ and $\exp (b / k)$ is that the temperature dependence of the vibrational frequencies is ignored in the latter. The variation from crystal to crystal of this temperature dependence offsets the variation in the coefficient $b$.

\subsection{Compensation}

Other empirical connections between pre-exponential factors and activation enthalpies have been noted in a wide variety of rate processes. These are usually described as compensation rules or Meyer-Neldel (1937) rules. The type of compensation involved is illustrated by the convergence shown in figure 2(c). Experimentally, it has been observed that, for related systems, plots of rates against temperature converge to a point at some temperature $T^{*}$, the compensation temperature (see Coutts and Pearsall (1984), Dosdale and Brook (1978), Dewsberry (1976), Eror and Wagner (1969), Hansel (1971), Irsigler et al (1983), Kemery and Rosenberg (1970), Metselaar and Oversluizer (1984), Meyer and Neldel (1937), Nespurek et al (1976), Peacock-Lopez and Suhl (1982), Roberts 1981, Roberts et al (1980) and Weichman and Kuzel (1970) and references therein). In figure 3(a) the temperature range of figure $2(c)$ is extended and, while the lines do not converge to a point, a high degree of compensation is found. Suppose that a common value $g^{*}$ has been found at temperature $T^{*}$, then from the relation

$$
g_{\mathrm{m}, p}(T)=h_{\mathrm{m}, p}-T s_{\mathrm{m}, p}
$$

we have

$$
s_{\mathrm{m}, p}=\left(h_{\mathrm{m}, p}-g^{*}\right) / T^{*}
$$

and hence

$$
\ln A=\ln \nu_{0}+s_{\mathrm{m}, p} / k=h_{\mathrm{m}, p} / k T^{*}+\left(\ln \nu_{0}-g^{*} / k T^{*}\right) .
$$

Therefore a plot for the family of $\ln A$ versus $h_{\mathrm{m}, p}$ should be linear; this is the usual form of the Meyer-Neldel rule. We have plotted our results in this way in figure $3(b)$ and find that the rule is approximately satisfied, with $\mathrm{MgO}$ showing some deviation, as is to be expected from figure $3(a)$. As mentioned in $\S 1$, we have deliberately chosen a family 

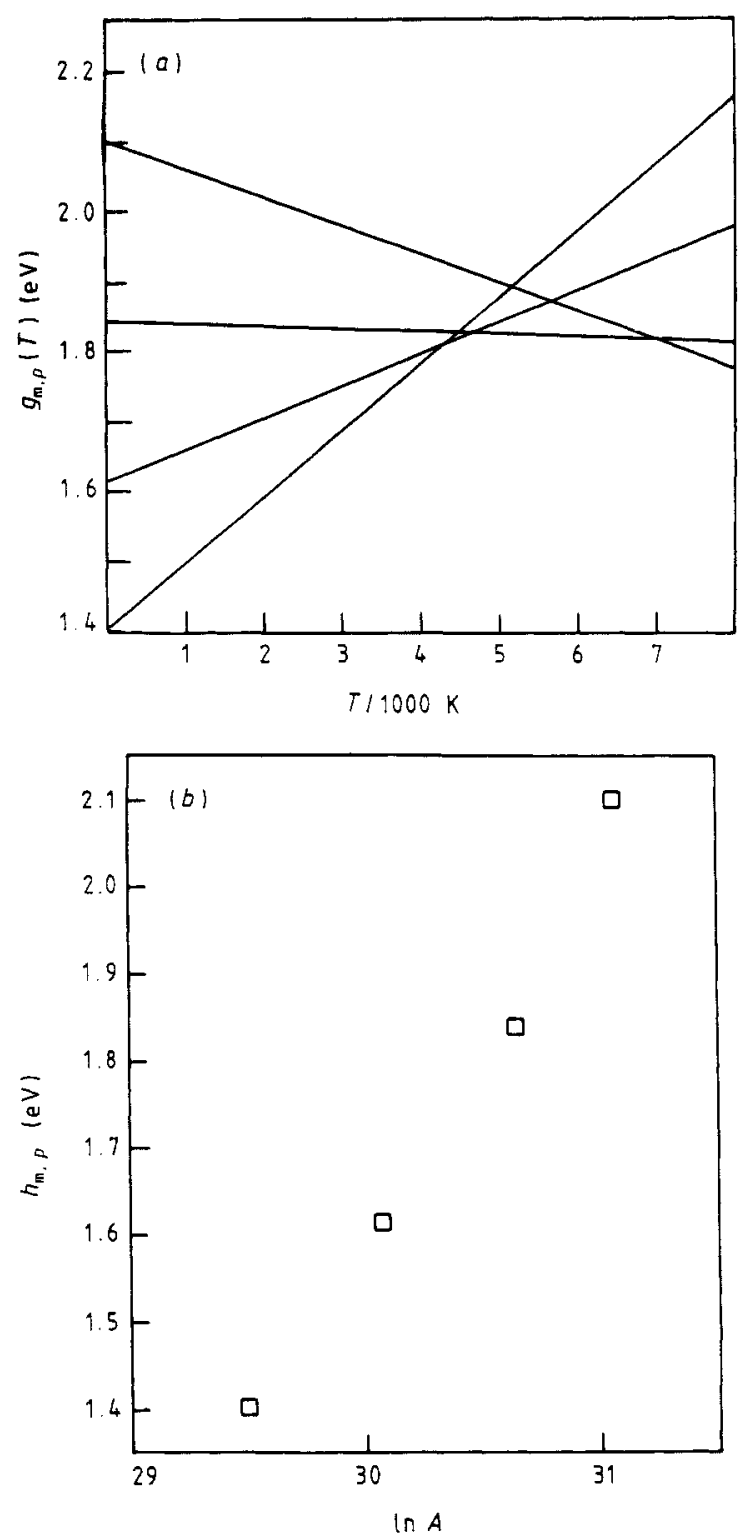

Figure 3. (a) Linear fits to $g_{m, p}(T)$ over an extended range of temperature; $(b)$ constantpressure migration enthalpies $h_{\mathrm{m}, p}$ versus $\ln A$ where $A$ is the pre-exponential frequency factor (if a Meyer-Neldel rule were satisfied, these points would lie in a straight line).

of host crystals which provides a good spread in both migration enthalpies and preexponential factors.

The scaling argument presented earlier in $\S 4.2$, while producing numerical results which are in good broad agreement with both our detailed calculations and the MeyerNeldel rule, provides no direct rationale for the rule. It is clear that any extension of the scaling argument to re-express it in the form of the Meyer-Neldel rule would require further connections between the energy, lattice parameter and cation mass ratios. 


\section{Concluding remarks}

Our discussion has indicated that, for a family of related systems, detailed calculations of the vibrational entropy terms for one member provide reasonable estimates for the other members. Since these calculations require considerable computer time, it is of real value to have some confidence in such estimates.

Clearly our present results do not allow us to explain the generality of the phenomenon of compensation. Nevertheless, the underlying reason here is that lattice expansion tends to lower both internal energies and vibrational frequencies, and large changes in one often correlate with changes in the other.

(C) 1987 UKAEA.

\section{References}

Catlow C R A, Corish J, Jacobs P W M and Lidiard A B 1981 J. Phys. C: Solid State Phys. 14 L121

Catlow C R A and Mackrodt W C (ed.) 1982 Computational Simulation of Solids, Springer Lecture Notes in Physics vol 166 (New York: Springer)

Coutts T and Pearsall N M 1984 Appl. Phys. Lett. 44134

Dewsberry R 1976 J. Phys. D: Appl. Phys. 9265

Dosdale T and Brook R T 1978 J. Mater. Sci. 13167

Eror N G and Wagner J B 1969 Phys. Status Solidi 35641

Flynn C P 1972 Point Defects and Diffusion (London: Oxford University Press)

Gillan M J and Jacobs P W M 1983 Phys. Rev. B 28759

Hansel H 1971 Ann. Phys., Paris 24147

Harding J H 1985a Physica A 13113

1985b Phys. Rev. B 326861

Irsigler P, Wagner D and Dunstan D J 1983 J. Phys. C: Solid State Phys. 166605

Kemery E and Rosenberg B 1970 J. Chem. Phys. 533549

Metselaar R and Oversluizer G 1984 J. Solid State Chem. 55320

Meyer W and Neldel H 1937 Z. Tekh. Phys. 18588

Mott N F and Littleton M J 1938 Trans. Faraday Soc. 34485

Nespurek S, Sworakowski J and Williams J O 1976 J. Phys. C: Solid State Phys. 92073

Norgett M J 1974 Atomic Energy Research Establishment Harwell Report R7650

1977 Atomic Energy Research Establishment Harwell Report M2880

Peacock-Lopez E and Suhl H 1982 Phys. Rev. B 263774

Roberts G G 1971 J. Phys. C: Solid State Phys. 43167

Roberts G G, Appsley N and Munn R W 1980 Phys. Rep. 6059

Sangster M J L and Stoneham A M 1984 J. Phys. C: Solid State Phys. 176093

Sempolinski D R and Kingery W D 1980 J. Am. Ceram. Soc. $\mathbf{6 3} 11$

Stoneham A M and Sangster M J L 1985 Phil. Mag. B 52717

Vineyard G H 1957 J. Phys. Chem. Solids 3121

Weichman F L and Kuzel 1970 Can. J. Phys. 4863 